\title{
Naturally occurring anti-glycan antibodies binding to Globo H-expressing cells identify ovarian cancer patients
}

Tatiana Pochechueva ${ }^{1 \dagger}$, Shahidul Alam ${ }^{1,2 \dagger}$, Andreas Schötzau' ${ }^{1}$ Alexander Chinarev ${ }^{3}$, Nicolai V. Bovin ${ }^{3}$, Neville F. Hacker ${ }^{4}$, Francis Jaco $b^{1,2^{*}}$ and Viola Heinzelmann-Schwarz ${ }^{1,5^{*}}$

\begin{abstract}
Background: Glycosphingolipids are important compounds of the plasma membrane of mammalian cells and a number of them have been associated with malignant transformation and progression, reinforcing tumour aggressiveness and metastasis. Here we investigated the levels of naturally occurring anti-glycan antibodies to Globo H in blood plasma obtained from high-grade serous ovarian cancer patients (SOC) and women without gynaecological malignancies (control) using suspension glycan array technology employing chemically synthesized glycans as antibody targets.

Results: We found that anti-human Globo H IgG antibodies were able to significantly discriminate SOC from controls $(P<0.05)$. A combination with the clinically used tumour marker CA125 increased the diagnostic performance (AUC 0.8711). We next compared suspension array with standard flow cytometry in plasma samples and found that the level of anti-Globo $\mathrm{H}$ antibodies highly correlated $(r=0.992)$. The incubation of plasma-derived anti-glycan antibodies with chemically synthesized (presented on fluorescence microspheres) and native Globo $\mathrm{H}$ (expressed on Globo H-positive cell lines) revealed strong reactivity of naturally occurring human anti-Globo H antibodies towards its antigen expressed on ovarian cancer cells.
\end{abstract}

Conclusions: Our data demonstrate that human plasma-derived antibodies to Globo $\mathrm{H}$ as well as the presence of the antigen might be considered as therapeutic option in ovarian cancer.

Keywords: Ovarian cancer, Glycosphingolipids, Suspension array, Glycan

\section{Background}

Globo $\mathrm{H}$ is a glycosphingolipid of the globo series with a sugar terminus resembling the blood group antigen $\mathrm{H}$ determinant. First identified in human teratocarcinoma and breast cancer cells $[1,2]$, Globo $\mathrm{H}$ was found to be overexpressed on the cell surface of several epithelial cancers such as breast, colon, endometrial, gastric, pancreatic, lung, and prostate cancers [3-5]. It is also moderately expressed in normal epithelial tissues (lung, breast, prostate, stomach, pancreas, and ovary), but its distribution is restricted to apical epithelial cells at lumen borders [5]. Functionally, Globo $\mathrm{H}$ has been

\footnotetext{
* Correspondence: francis.jacob@unibas.ch; Viola.Heinzelmann@usb.ch ${ }^{\dagger}$ Equal contributors

'Ovarian Cancer Research, Department of Biomedicine, University Hospital Basel, University of Basel, Basel, Switzerland

Full list of author information is available at the end of the article
}

associated with tumour stem cells [6], to be a potent inducer of angiogenesis [7], and an immunosuppressor through Notch signalling [8]. The high Globo $\mathrm{H}$ expression by only cancer and cancer stem cells made it an attractive target for generation of therapeutic cancer vaccines. These vaccines underwent the long history of development and improvement and are currently being tested in clinical trials for treatment [9-11]. Globo $\mathrm{H}$ has therefore been considered as one promising tumorassociated glycan biomarker, in particular for breast cancer.

Plasma-derived naturally occurring anti-glycan antibodies (AGA) have been associated to various human diseases including cystic fibrosis [12], Crohn's disease [13], and various malignancies [14-18]. Also healthy humans harbour sets of AGA to blood group-, xeno- 
(heterophil), and infection-related glycan motifs [19]. Globo $\mathrm{H}$ was also shown to be an antigen for naturally occurring plasma-derived AGA in breast and some other epithelial cancers with high specificity [20]. These antibodies were assessed by the glycan microarray technology and their levels were significantly different in serum of breast cancer patients and healthy individuals [21]. Despite these few reports, there is limited knowledge on Globo $\mathrm{H}$ antigen expression on cell lines and the presence of naturally circulating AGA in plasma from ovarian cancer patients.

We assessed the levels of anti-Globo $\mathrm{H}$ antibodies of $\operatorname{IgG}$ and $\operatorname{IgM}$ isotypes in healthy and serous ovarian cancer (SOC) patients. We used our "in house" developed suspension glycan array (SGA), a robust tool for AGA profiling $[15,22]$, to detect anti-Globo $\mathrm{H}$ antibodies in human plasma samples for discrimination of ovarian cancer patients and normal controls. We also compared SGA and standard flow cytometry in order to address the following questions; a) whether AGA levels are comparable in both methods, and b) whether those antibodies can recognize native Globo $\mathrm{H}$ epitope on the cell surface of cancer cell lines.

\section{Methods}

\section{Human subjects}

Two groups of female donors were involved in the current study. All patients were over the age of 18 and were prospectively included after giving informed consent in accordance with ethical regulations (Hunter Area Research Ethics 04/04/07/3.04; South Eastern Sydney Illawarra HREC/AURED Ref:08/09/17/3.02). The serous ovarian cancer (SOC) group consisted from patients with serous ovarian cancer with Grade 2 and 3 of all FIGO stages (SOC $n=19)$. The control group $(n=29)$ consisted of women without macroscopically confirmed gynaecological malignancy including comprising of benign donors, patients with benign gynaecological tumours (leiomyoma, cystadenoma, or fibroadenoma) and patients undergoing medical control due to previous family history of breast cancer or bearing known BRCA mutations. Women in the control group had no other type of malignancy at the date of collection. Patients were either admitted with an adnexal mass to the Gynaecological Cancer Centre of the Royal Hospital for Women, Randwick, Australia or were seen as outpatients to the Hereditary Cancer Centre of The Prince of Wales Hospital, Randwick, Australia. The processing of blood plasma samples was performed as previously described $[15,23]$.

\section{Suspension glycan array}

Glyco-polymers with end-biotin group- Sialylated and sulfated glycans were chemically synthesised (Lectinity
Holdings, Moscow, Russia. The glycopolymers, Glyc(20)PAA-biot ${ }_{1}$, used for coupling to fluorescent beads were produced in-house as previously described [24]. The glycopolymers are composed of a polyacrylamide carrier (PAA, number of the average polymerisation degree, $n=$ 220) provided with end biotin groups and side-pendant Glyc residues, that are statistically distributed along the polymer backbone. The content of monomer units with glycan substitution is $20 \mathrm{~mol} \%$. Non-glycosylated monomer units are substituted with an ethanolamine residue for the regular Glyc(20)-PAA-biot ${ }_{1} . \mathrm{GD}_{3}$ and $\mathrm{GM}_{2}$ glycans were purchased from Elicityl (Crolles, France) and then used for synthesis of Glyc(20)-PAA-biot ${ }_{1}$, as described above.

Modification of fluorescent microbeads- Biotinylated glycopolymers were coupled to fluorescent Bio-Plex Pro $^{\text {Tx }}$ Magnetic $\mathrm{COOH}$ beads of $6.5 \mu \mathrm{m}$ diameter with distinct spectral "addresses" (Bio-Rad Laboratories Inc., Cressier, Switzerland). Each bead's region was embedded with a precise ratio of red and infrared fluorescent dyes allowing its identification using Bio-Plex 200 Suspension Array System (Bio-Rad Laboratories Inc., Hercules, CA, USA). Coupling of biotinylated glycopolymers using biot$\mathrm{PEG}_{23}-\mathrm{NH}_{2}$ (Hetero-bifunctional PEG, $\mathrm{MW}=1300$, Iris Biotech $\mathrm{GmbH}$, Marktredwitz, Germany) modification of microbeads was accomplished as described previously [25]. All remaining steps were performed as described previously [25]. Data are presented as median MFI and interquartile range (IQR).

\section{ELISA}

CA125 levels in blood samples obtained from patients were measured using the human CA125 ELISA kit (MUC16) SimpleStep (Abcam, LucernaChem AG, Switzerland, \#ab195213). ELISA was performed according to manufacture's protocol.

\section{Cell culture}

Ovarian (IGROV1, A2780, SKOV3, BG1, OVCAR3, OVCAR4, OVCAR5, OVCAR8, OAW42, TYK-nu, KURAMUCHI, OVSAHO, CAOV3, and TOV21G), and breast (T47D and MCF7) cancer cell lines, ovarian surface epithelial cells (HOSE17.1 and HOSE6.3) and normal mammary epithelial cell line MCF10A were grown in RPMI-1640 media (Sigma-Aldrich, Buchs, Switzerland) supplemented with $10 \%$ fetal bovine serum (FBS), penicillin $(100 \mathrm{U} / \mathrm{ml})$ and streptomycin $(100 \mu \mathrm{g} / \mathrm{ml})$ (obtained from Sigma-Aldrich). Fallopian tube secretory epithelial cells (FT237 and FT240) were grown in DMEM-Ham's F12 medium without HEPES buffer (Sigma) supplemented with Ultroser ${ }^{\mathrm{Tm}}$ (PALL corporation, Life Science, Switzerland), penicillin $(100 \mathrm{U} / \mathrm{ml})$ and streptomycin $(100 \mu \mathrm{g} / \mathrm{ml})$. Another ovarian cancer cell line, EFO27 were grown in RPMI-1640 media supplemented with 20\% FBS, penicillin $(100 \mathrm{U} / \mathrm{ml})$, streptomycin $(100 \mu \mathrm{g} / \mathrm{ml})$ and $1 \mathrm{mM}$ 
sodium pyruvate (purchased from Sigma). The culture condition for all cell lines were $37^{\circ} \mathrm{C}$ in a $95 \%$ humidified atmosphere containing $5 \% \mathrm{CO}_{2}$.

\section{Flow cytometry}

Cells were seeded in $\mathrm{T} 25 \mathrm{~cm}^{2}$ flask cultivated to $70-80 \%$ confluence, washed with PBS, harvested with $1 \mathrm{x}$ nonenzymatic cell dissociation solution at $37{ }^{\circ} \mathrm{C}$ and counted using trypan blue. Cells were than transferred to 96well- V-bottom micro test plate $\left(10^{5}\right.$ cells/well $)$ and pelletized at 400xg for $5 \mathrm{~min}$. Analysis of cell membranebound Globo $\mathrm{H}$ was performed with two-step staining procedure. Anti-Globo H antibodies (Mbr1_IgM_1:500, diluted in FACSWASH (1\% BSA in PBS)) were added to cells, resuspended and incubated on ice for $1 \mathrm{~h}$. After two washes with FACSWASH, cells were incubated with R-PE (1:200) on ice for 30 min. Following two washing steps, cells were incubated with 7-AAD (1:100) on ice for $10 \mathrm{~min}$. After the final wash, cells were resuspended in $200 \mu \mathrm{l}$ of FACSWASH and analyzed with BD Accuri C6 flow cytometer (BD Pharmingen). Analysis of inhibition experiments using microspheres and human cell lines were analyzed with BD LSR Fortessa. Data analysis was done using FlowJo v10.0.4 (Tree Star Inc., Ashland, USA).

\section{Statistical analysis}

The raw data (obtained as MFI) were log-transformed. Statistical analysis was either performed by GraphPad Prism 6 software or the statistical software $R$ version 3.1.3 (www.R-project.org). Penalized logistic regression and receiver operating characteristics (ROC) analysis was performed using the R packages 'glmnet' and 'ROCR' [26], respectively. Comparisons between subgroups were performed using Mann Whitney $U$-test. A $p$ value $<0.05$ was considered significant.

\section{Results and discussion}

Studies in the past suggested that levels of anti-Globo $\mathrm{H}$ antibodies are predictive for breast cancer [20, 21]. Recent genomic and transcriptomic analyses by the Cancer Genome Atlas (TCGA) revealed that basal-like breast cancer displays significant molecular similarities to undifferentiated (high-grade) SOC [27, 28]: both diseases share molecular signatures (e.g. mutations in TP53, BRCA1/2, and RB1). Thus, we investigated the presence of AGA to Globo $\mathrm{H}$ in a cohort of 48 blood plasma samples, consisting of high-grade SOC $(n=19)$ and a control group $(n=29)$ comprising women without or benign disease of the gynaecological tract (e.g. leiomyoma, cystadenoma, or fibroadenoma).

The SGA utilized to measure AGA [22, 25] incorporated coupling of microspheres with an internal fluorescence to chemically synthesized glycans (Fig. 1a) via a linker (Fig. 1b). Using monoplexed SGA levels of antibodies to alpha-rhamnose (positive control) and Globo $\mathrm{H}$ were generally high for both isotypes in all samples (Fig. 1c-e). In contrast, aminoglucitol (negative control) showed AGA levels below the threshold (Fig. 1c). We observed significantly decreased anti-globo $\mathrm{H}$ antibody levels of IgG isotype in SOC patients to the control group (IgG 2642 MFI (338; 6067), $p=0.009$, Fig. 1d-e), hence clearly discriminating cancer patients from healthy controls. The comparison for the IgM isotype showed a similar trend (1526 (492; 4154), $p=0.071$, Fig. 1d-e). We next performed ROC analysis including the current clinical ovarian cancer biomarker CA125, which was measured by ELISA during clinical examinations at initial diagnosis. The combination of antiGlobo $\mathrm{H}$ IgG and IgM revealed an area under the curve (AUC) of 0.7441 . The addition of CA125 in a penalized logistic regression model increased the AUC to 0.8711 , which was even higher than CA125 alone (AUC 0.8539). This indicates that anti-globo $\mathrm{H}$ antibody levels improve the diagnostic performance of CA125 alone (Fig. 1f).

Despite this intriguing finding it remained to be elucidated whether anti-Globo $\mathrm{H}$ antibodies are capable to bind the native Globo $\mathrm{H}$ antigen expressed on ovarian cancer cells and whether plasma-derived AGA also recognize other glycan structures (epitopes similar to Globo H). Thus, we profiled a panel of ovarian $(n=15)$ and breast $(n=2$, used as positive control, [11]) cancer cells (Fig. 2a) using the monoclonal antibody MBr1 (Fig. 2a). After the evaluation of the MBr1 in breast cancer cell lines MCF7 and T47D, the working concentration $2.5 \mu \mathrm{g} / \mathrm{ml} \mathrm{MBr1}$ was selected. We observed Globo $\mathrm{H}$ expression in only three ovarian cancer cell lines, OVCAR3, BG1, and IGROV1 (Fig. 2a). The remaining ovarian cancer cell lines never showed any positivity for Globo $\mathrm{H}$. The normal ovarian surface epithelial cell lines (HOSE17-1 and HOSE6-3), fallopian tube secretory epithelial cells (FT237 and FT240), and the mammary epithelial cell line MCF10A were also negative for Globo $\mathrm{H}$ (Fig. 2a). In contrast to our findings, the literature reported Globo $\mathrm{H}$ expression also in TOV21G ovarian cancer cells [29]. This discrepancy might be explained by the use of different monoclonal antibodies to Globo $\mathrm{H}$ (MBr1 and VK9) in these two studies.

In order to study the binding of naturally occurring anti- Globo $\mathrm{H}$ antibodies from patient samples to Globo H-positive ovarian cancer cell lines, we compared SGA and standard flow cytometry. A total of fourteen plasma samples reflecting the spectrum of antibody levels were selected and profiled using both methods. The comparison revealed a high correlation $(r=0.992, p<0.0001)$ concluding that flow cytometry is suitable for AGA profiling (Fig. 2b). 


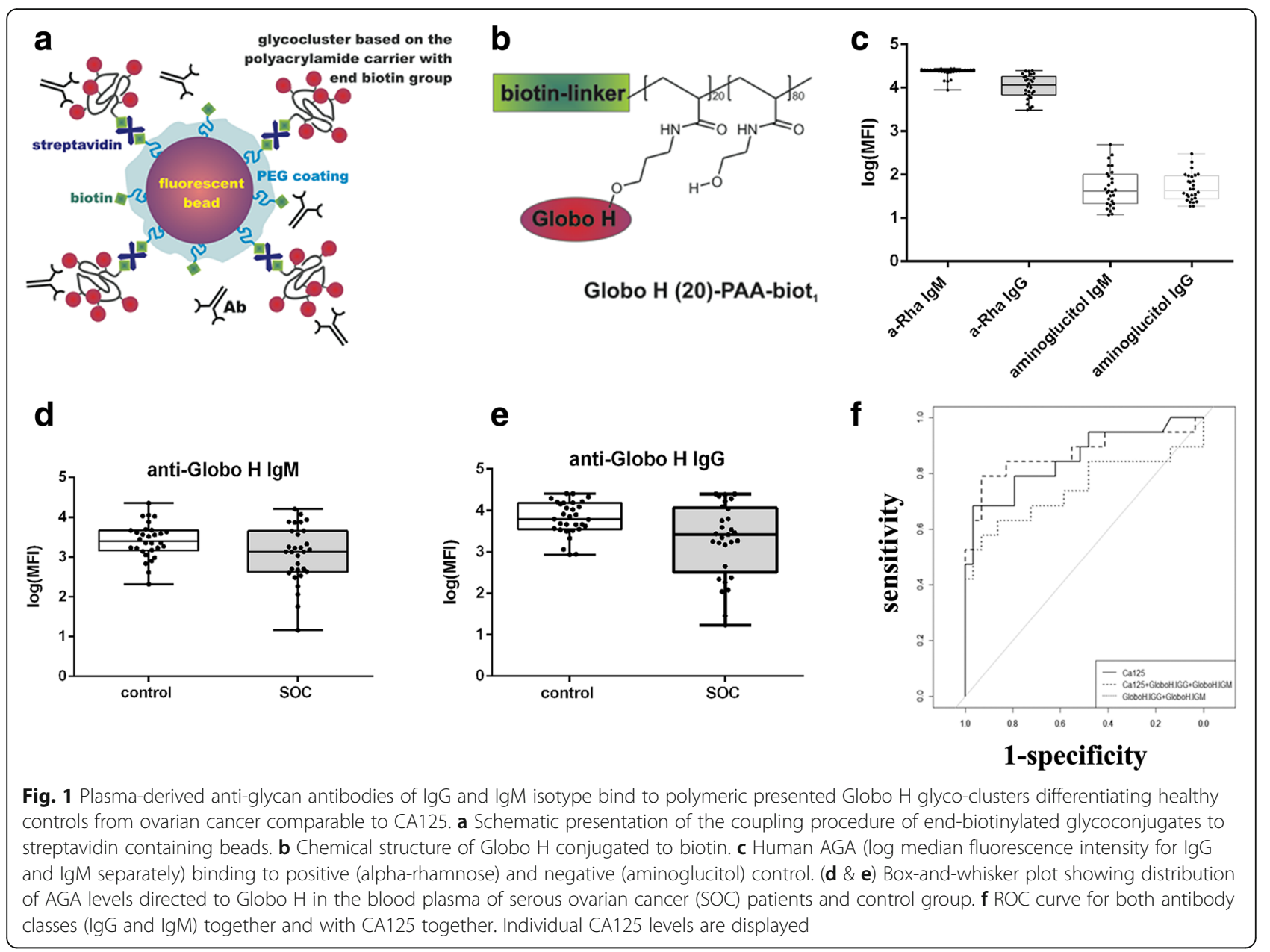

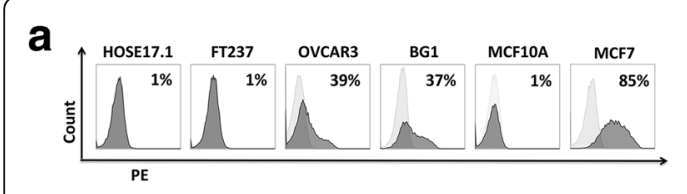

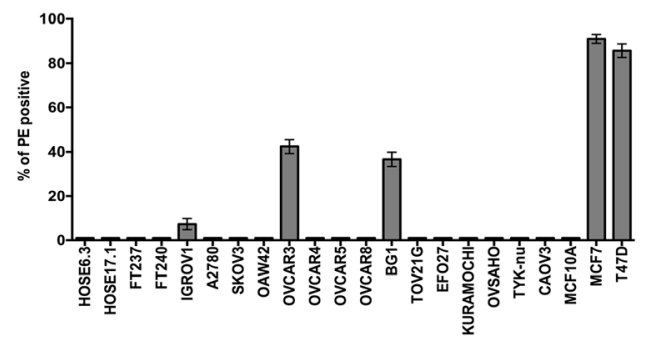

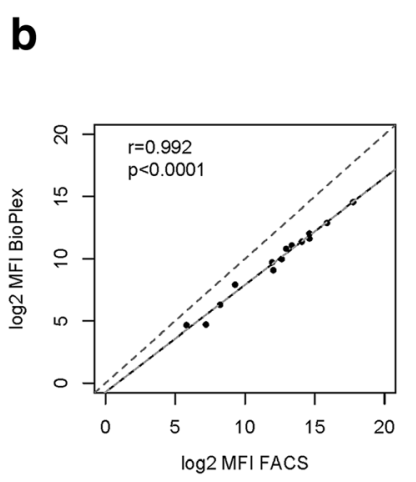

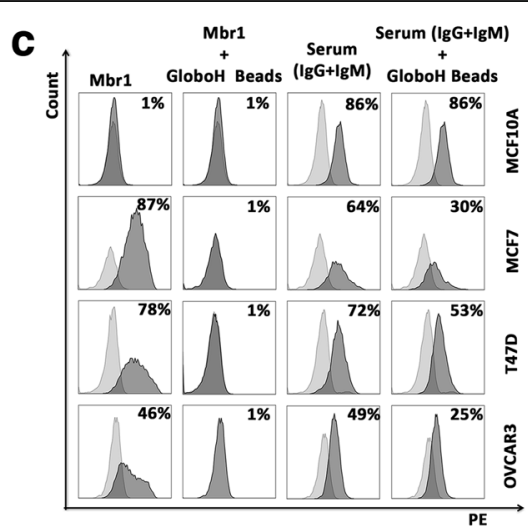

Fig. 2 Anti-Globo $\mathrm{H}$ antibodies bind ovarian cancer cell lines determined to express naturally synthesized Globo $\mathrm{H}$ on the cell surface membrane. a Flow cytometry results showing expression of Globo $\mathrm{H}$ in selected breast and ovarian cancer cell lines. Representative histograms for cell lines HOSE17.1, FT237, OVCAR3, BG1, MCF10A and MCF7. The percentages of positively stained cells were displayed and are a representative of five independent experiments. Bar chart summarizing five independent experiments on all cell lines tested showing the mean and standard deviation for each cell line. $\mathbf{b}$ SGA and FACS show high correlation for binding of plasma-derived anti-Globo H antibodies to Globo H-coupled microbeads (SGA) and Globo-H positive cells (FACS, here MCF7 cells); plasma samples 1-14, dilutions 1/40 (SGA) and 1/200 (FACS). MFI values were log-transformed. c Flow cytometry data represented as histogram for negative control (light grey) and positively stained sample (dark grey). The value provided in each histogram refers to the percentage of positively stained cells 
To finally prove that plasma antibodies indeed bind to Globo $\mathrm{H}$ on the cell surface, we co-incubated anti-Globo $\mathrm{H}$ positive plasma (a pool of five individual samples) with Globo $\mathrm{H}$-coupled microbeads $\left(10^{5}\right.$ beads). As a negative control we used MCF10A. We found that plasma antibody binding decreased to different extents among all Globo $\mathrm{H}$ positive cancer cell lines (MCF7, T47D, and OVCAR3) tested in flow cytometry (Fig. 2c). We did not achieve pronounced decrease of binding using plasma dilution 1/5-1/100 as well as with combination of less than $0.5 \times 10^{5}$ beads. The plasma binding in case of MCF10A was high (86\%) and did not decrease after plasma pre-incubation with Globo H beads (Fig. 2c). These data clearly demonstrate that ovarian cancer cells express Globo $\mathrm{H}$ and that the antigen is recognized by naturally occurring anti-Globo $\mathrm{H}$ antibodies from human blood plasma.

Taken together, we demonstrate that women with SOC and benign disease display different levels of anti-Globo $\mathrm{H}$ antibodies. Moreover, these antibodies are capable to bind Globo $\mathrm{H}$ expressing ovarian cancer cell lines.

\section{Conclusions}

In this study we report on naturally occurring AGA to a well-known tumour-associated carbohydrate antigen (Globo $\mathrm{H}$ ) in ovarian cancer. The current study is based on Globo $\mathrm{H}$ identified by circulating AGA as well as the monoclonal antibody $\mathrm{MBr}$. However, future studies have to consider larger cohorts and the measurement of combinations with other representative biomarkers including AGA to evaluate the diagnostic performance of Globo H. Further studies might also incorporate mass spectrometry to confirm the presence of the antigen in ovarian cancer cells. Verification in tissue samples might also support a potential role of Globo $\mathrm{H}$ in this malignant disease, e.g. by MALDI imaging mass spectrometry [30]. This knowledge might also contribute to our understanding of the functional role of Globo $\mathrm{H}$ as it has been associated with cancer stem (-like) cells previously [31].

\section{Abbreviations}

AGA: Anti-glycan antibodies; AUC: Area under the curve; FT: Fallopian tube; FBS: Fetal bovine serum; HOSE: Human ovarian surface epithelium; MFI: Median fluorescence intensity; ROC: Receiver operating characteristics; SOC: Serous ovarian cancer; SGA: Suspension glycan array; TCGA: The cancer genome atlas

\section{Acknowledgements}

We are grateful to the Flow Cytometry Facility at the Department of Biomedicine, University Hospital Basel and University of Basel (Danny Labes and Emmanuel Traunecker) for providing all necessary support.

\section{Funding}

Swiss National Foundation (310030 143619 and 32) to V.H.S., Oncosuisse Grant (KFS_3013-08-2012 to V.H.S.), and Krebsliga beider Basel 06-2013/14-2015 to V.H.S and F.., respectively, and the Department of Biomedicine, University Hospital Basel, University of Basel.
Availability of data and materials

All data generated or analysed during this study are included in this published article.

\section{Authors' contributions}

TP, AS, and FJ performed the experiments (suspension array, cell culture, flow cytometry, and competition assay). ASch carried out the statistical analysis. AC and NVB designed, synthesized and provided glycan structures. VHS carried out ethical approval, patient recruitment, and collection of material. NFH participated in cases recruitment of the present study. VHS and FJ designed the study. TP, AS, FJ, and VHS drafted the manuscript. All authors read and approved the final manuscript.

\section{Competing interests}

The authors declare that they have no competing interests.

\section{Consent for publication}

Not applicable.

Ethics approval and consent to participate

The Hunter Area Research Ethics (04/04/07/3.04) and South Eastern Sydney Illawarra HREC/AURED (08/09/17/3.02) approved this study.

\section{Author details}

'Ovarian Cancer Research, Department of Biomedicine, University Hospital Basel, University of Basel, Basel, Switzerland. ${ }^{2}$ Glyco-Oncology, Ovarian Cancer Research, Department of Biomedicine, University Hospital Basel, University of Basel, Basel, Switzerland. ${ }^{3}$ Shemyakin- Ovchinnikov Institute of Bioorganic Chemistry, Russian Academy of Sciences, 117997 Moscow, Russian

Federation. ${ }^{4}$ Royal Hospital for Women, Gynecological Cancer Centre, School of Women's and Children's Health, University of New South Wales, Sydney, Australia. ${ }^{5}$ Hospital for Women, Department of Gynecology and Gynaecological Oncology, University Hospital Basel, Basel, Switzerland.

Received: 22 October 2016 Accepted: 4 February 2017

Published online: 10 February 2017

\section{References}

1. Menard S, Tagliabue E, Canevari S, Fossati G, Colnaghi MI. Generation of monoclonal antibodies reacting with normal and cancer cells of human breast. Cancer Res. 1983;43(3):1295-300.

2. Kannagi R, Levery SB, Ishigami F, Hakomori S, Shevinsky LH, Knowles BB, et al. New globoseries glycosphingolipids in human teratocarcinoma reactive with the monoclonal antibody directed to a developmentally regulated antigen, stage-specific embryonic antigen 3. J Biol Chem. 1983:258(14):8934-42.

3. Bremer EG, Levery SB, Sonnino S, Ghidoni R, Canevari S, Kannagi R, et al. Characterization of a glycosphingolipid antigen defined by the monoclonal antibody $\mathrm{MBr} 1$ expressed in normal and neoplastic epithelial cells of human mammary gland. J Biol Chem. 1984;259(23):14773-7.

4. Canevari S, Fossati G, Balsari A, Sonnino S, Colnaghi MI. Immunochemical analysis of the determinant recognized by a monoclonal antibody (MBr1) which specifically binds to human mammary epithelial cells. Cancer Res. 1983;43(3):1301-5

5. Zhang S, Cordon-Cardo C, Zhang HS, Reuter VE, Adluri S, Hamilton WB, et al. Selection of tumor antigens as targets for immune attack using immunohistochemistry: I. Focus on gangliosides. Int J Cancer. 1997; 73(1):42-9.

6. Chang WW, Lee CH, Lee P, Lin J, Hsu CW, Hung JT, et al. Expression of Globo $\mathrm{H}$ and SSEA3 in breast cancer stem cells and the involvement of fucosyl transferases 1 and 2 in Globo H synthesis. Proc Natl Acad Sci U S A. 2008;105(33):11667-72. doi:10.1073/pnas.0804979105.

7. Cheng JY, Wang SH, Lin J, Tsai YC, Yu J, Wu JC, et al. Globo-H ceramide shed from cancer cells triggers translin-associated factor X-dependent angiogenesis. Cancer Res. 2014;74(23):6856-66. doi:10.1158/0008-5472.CAN-14-1651.

8. Tsai YCHJ, Cheng JY, Lin JJ, Hung JT, Wu YY, Yen KT, Yu AL. A prevalent cancer associated glycan, Globo $\mathrm{H}$ ceramide, induces immunosupression by reducing Notch 1 signalling. J Cancer Sci Ther. 2013;5(7):264-70.

9. Slovin SF, Ragupathi G, Fernandez C, Jefferson MP, Diani M, Wilton AS, et al. A bivalent conjugate vaccine in the treatment of biochemically relapsed 
prostate cancer: a study of glycosylated MUC-2-KLH and Globo H-KLH conjugate vaccines given with the new semi-synthetic saponin immunological adjuvant GPI-0100 OR QS-21. Vaccine. 2005;23(24):3114-22. doi:10.1016/j.vaccine.2005.01.072.

10. Danishefsky SJ, Shue YK, Chang MN, Wong CH. Development of globo-h cancer vaccine. Acc Chem Res. 2015;48(3):643-52. doi:10.1021/ar5004187.

11. Huang YL, Hung JT, Cheung SK, Lee HY, Chu KC, Li ST, et al. Carbohydratebased vaccines with a glycolipid adjuvant for breast cancer. Proc Natl Acad Sci U S A. 2013;110(7):2517-22. doi:10.1073/pnas.1222649110.

12. Hirche TO, Stein J, Hirche H, Hausmann J, Wagner TO, Behrens F, et al. Increased levels of anti-glycan antibodies in patients with cystic fibrosis. Eur J Med Res. 2011;16(9):385-90.

13. Rieder F, Lopez R, Franke A, Wolf A, Schleder S, Dirmeier A, et al. Characterization of changes in serum anti-glycan antibodies in Crohn's disease - a longitudinal analysis. PLoS One. 2011;6(5):e18172.

14. Jacob F, Anugraham M, Pochechueva T, Tse BW, Alam S, Guertler R, et al. The glycosphingolipid $P(1)$ is an ovarian cancer-associated carbohydrate antigen involved in migration. Br J Cancer. 2014;111(8): 1634-45. doi:10.1038/bjc.2014.455.

15. Jacob F, Goldstein DR, Bovin NV, Pochechueva T, Spengler M, Caduff R, et al. Serum antiglycan antibody detection of nonmucinous ovarian cancers by using a printed glycan array. Int J Cancer. 2012;130(1):138-46. doi:10.1002/ ijc.26002.

16. Pochechueva T, Jacob F, Goldstein DR, Huflejt ME, Chinarev A, Caduff R, et al. Comparison of printed glycan array, suspension array and ELISA in the detection of human anti-glycan antibodies. Glycoconj J. 2011; 28(8-9):507-17.

17. Pedersen JW, Blixt O, Bennett EP, Tarp MA, Dar I, Mandel U, et al. Seromic profiling of colorectal cancer patients with novel glycopeptide microarray. Int J Cancer. 2011;128(8):1860-71. doi:10.1002/ijc.25778.

18. Wandall HH, Blixt O, Tarp MA, Pedersen JW, Bennett EP, Mandel U, et al. Cancer biomarkers defined by autoantibody signatures to aberrant Oglycopeptide epitopes. Cancer Res. 2010;70(4):1306-13.

19. Huflejt ME, Vuskovic M, Vasiliu D, Xu H, Obukhova P, Shilova N, et al. Anticarbohydrate antibodies of normal sera: findings, surprises and challenges. Mol Immunol. 2009;46(15):3037-49.

20. Huang CY, Thayer DA, Chang AY, Best MD, Hoffmann J, Head S, et al. Carbohydrate microarray for profiling the antibodies interacting with Globo H tumor antigen. Proc Natl Acad Sci U S A. 2006;103(1):15-20. doi:10.1073/pnas.0509693102

21. Wang CC, Huang $Y L$, Ren $C T$, Lin CW, Hung JT, Yu JC, et al. Glycan microarray of $\mathrm{Globo} \mathrm{H}$ and related structures for quantitative analysis of breast cancer. Proc Natl Acad Sci U S A. 2008;105(33):11661-6. doi:10.1073/pnas.0804923105.

22. Pochechueva T, Chinarev A, Spengler M, Korchagina E, HeinzelmannSchwarz V, Bovin N, et al. Multiplex suspension array for human anti-carbohydrate antibody profiling. Analyst. 2011;136(3):560-9. doi:10.1039/c0an00758g.

23. Jacob F, Meier M, Caduff R, Goldstein D, Pochechueva T, Hacker N, et al. No benefit from combining HE4 and CA125 as ovarian tumor markers in a clinical setting. Gynecol Oncol. 2011;121(3):487-91. doi:10.1016/j.ygyno.2011.02.022.

24. Chinarev AA, Galanina OE, Bovin NV. Biotinylated multivalent glycoconjugates for surface coating. Methods Mol Biol. 2010;600:67-78.

25. Pochechueva T, Chinarev A, Bovin N, Fedier A, Jacob F, HeinzelmannSchwarz V. PEGylation of microbead surfaces reduces unspecific antibody binding in glycan-based suspension array. J Immunol Methods. 2014;412: 42-52. doi:10.1016/.jim.2014.06.015.

26. Sing T, Sander O, Beerenwinkel N, Lengauer T. ROCR: visualizing classifier performance in R. Bioinformatics. 2005;21 (20):3940-1.

27. Cancer Genome Atlas N. Comprehensive molecular portraits of human breast tumours. Nature. 2012;490(7418):61-70. doi:10.1038/nature11412.

28. Cancer Genome Atlas Research N. Integrated genomic analyses of ovarian carcinoma. Nature. 2011;474(7353):609-15. doi:10.1038/nature10166.

29. Lou YW, Wang PY, Yeh SC, Chuang PK, Li ST, Wu CY, et al. Stage-specific embryonic antigen-4 as a potential therapeutic target in glioblastoma multiforme and other cancers. Proc Natl Acad Sci U S A. 2014;111(7):2482-7. doi:10.1073/pnas. 1400283111.

30. Everest-Dass AV, Briggs MT, Kaur G, Oehler MK, Hoffmann P, Packer NH. $\mathrm{N}$-glycan MALDI Imaging Mass Spectrometry on Formalin-Fixed Paraffin-
Embedded Tissue Enables the Delineation of Ovarian Cancer Tissues. Mol Cell Proteomics. 2016;15(9):3003-16. doi:10.1074/mcp.M1 16.059816.

31. Liang YJ, Kuo HH, Lin CH, Chen YY, Yang BC, Cheng YY, et al. Switching of the core structures of glycosphingolipids from globoand lacto- to ganglio-series upon human embryonic stem cell differentiation. Proc Natl Acad Sci U S A. 2010;107(52):22564-9. doi:10.1073/pnas.1007290108

\section{Submit your next manuscript to BioMed Central and we will help you at every step:}

- We accept pre-submission inquiries

- Our selector tool helps you to find the most relevant journal

- We provide round the clock customer support

- Convenient online submission

- Thorough peer review

- Inclusion in PubMed and all major indexing services

- Maximum visibility for your research

Submit your manuscript at www.biomedcentral.com/submit
Biomed Central 